\title{
3D Finite Element Simulations of Strip Lines in a YBCO/Au Fault Current Limiter
}

\author{
J. Duron, L. Antognazza, M. Decroux, F. Grilli, S. Stavrev, B. Dutoit and Ø. Fischer
}

\begin{abstract}
Geometrical aspects of the design of fault current limiters (FCL) have a great impact on their performances. Recently, the University of Geneva have made certain optimizations by splitting the FCL into many small dissipative lengths in order to achieve a distributed transition along the device.

For this paper, we have performed new 3D finite element method (FEM) simulations for studying the behavior of strip lines of a YBCO/Au FCL in an AC nominal use (sinusoidal current at industrial frequency) up to $3 I_{c}$. The very large aspect ratio of the device needs a particular attention to the modeling and meshing process.

The numerical results show that presence of sharp corners can influence the performance of the device. Due to the high value of the electric field in these areas, the local losses are much higher than in the case of smooth corners, and this may lead to burning and cracking the wafer. Irreversible damage experiments have confirmed these locations.

In this paper we proposed new geometries, taking into account the length of the connecting path and the corners optimization in order to decrease the risk of very high localized losses in the meander.
\end{abstract}

Index Terms-Fault currents, superconducting devices, superconducting thin films, numerical modeling.

\section{INTRODUCTION}

Fault current limiters (FCLs) are considered to be one of the most promising applications of high temperature superconductors (HTS) since they have no counterpart in the conventional power equipment technology.

All thin-film based FCLs have a similar behavior: the switching time is very low (of the order of few microseconds), and the current peak during a short circuit reaches 3-4 times the critical current [1]. However, it has been observed that only a part of the FCL is actually limiting the current and is also supporting all the dissipated power [2]. To improve the performance of these FCLs, the University of Geneva has proposed a new design by splitting the initial dissipative area into many dissipative lengths [3].

The fault current limiter is composed of a YBCO thin film (thickness $300 \mathrm{~nm}$ ) covered by a gold layer $(25 \mathrm{~nm}$ or $125 \mathrm{~nm})$.

Manuscript received December 20, 2004. This work has been supported by the Swiss National Science Foundation through the National Center of Competence in Research "Materials with Novel Electronic Properties MaNEP".

J. Duron, F. Grilli, S. Stavrev, and B. Dutoit are with the Swiss Federal Institute of Technology - Lausanne, EPFL-IC-LANOS, 1015 Lausanne, Switzerland (e-mail: joseph.duron@epfl.ch).

F.Grilli is now with the Los Alamos National Laboratory, MST-STC, Los Alamos, New Mexico 87545, United States

L. Antognazza, M. Decroux and Ø. Fischer are with the University of Geneva - Geneva, DPMC, 1211 Geneva 4, Switzerland.

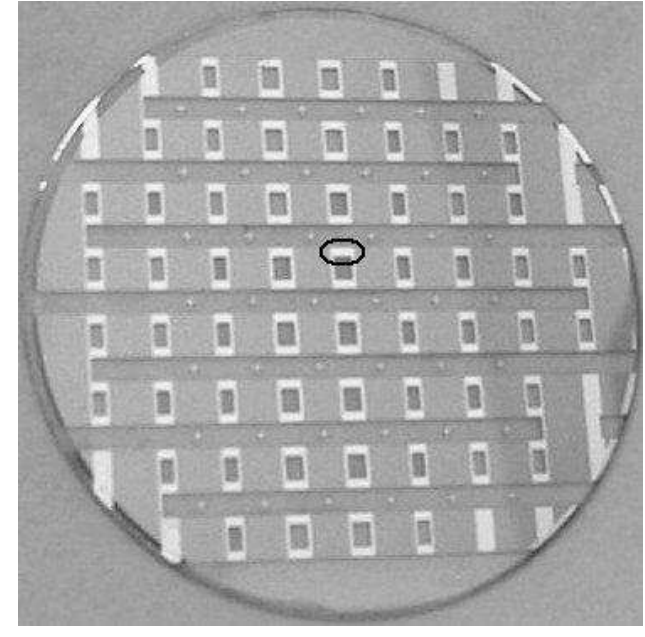

Fig. 1. FCL with novel geometry made by the University of Geneva. The black ellipse indicates the localization of the damage shown in Fig. 2

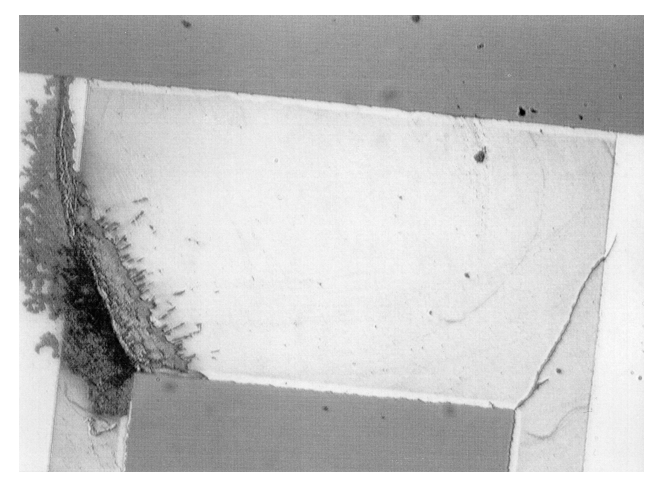

Fig. 2. Picture of a constriction showing damage after destructive experiments

This novel design is based on a local width reduction achieved by substituting the superconducting meander with consecutive constrictions, see Fig. 1, in order to accelerate the superconducting to normal state transition of the full wafer.

This new design leads to optimization of the system because, in case of fault, the power is more evenly distributed along the device. This has been experimentally confirmed on a $5 \mathrm{~kW}$ FCL on 2 inch wafer [3]. However, during severe tests, the materials have burned due to local high temperature values. In Fig. 2, we can see that the degradations seem to be localized in the constrictions' corners.

The aim of this paper is to verify the localization of the damage, to understand its origin and to propose solutions in order to avoid the burning of the device. 


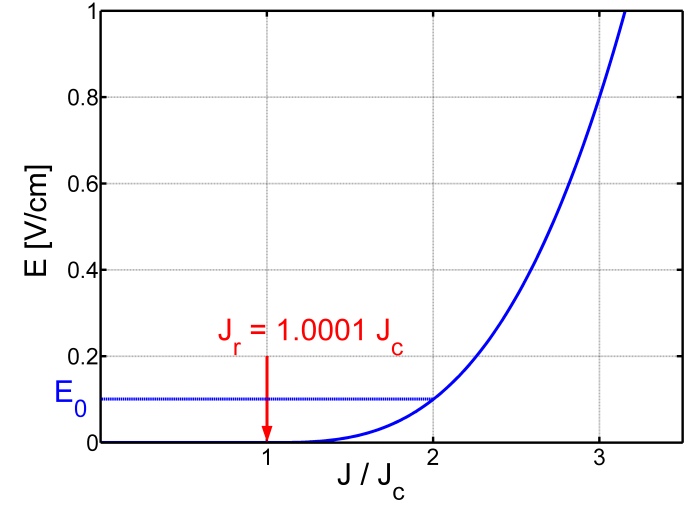

Fig. 3. $E-J$ characteristic modeled with equation (2)

\section{NUMERICAL SIMULATIONS}

For validating the hypothesis of localization of the damage at the constrictions' corners, new FEM simulations have been made with the software Flux 3D [4] in order to study the current density and electric field distribution in 3D inside the FCL. The group at the Swiss Federal Institute of Technology has demonstrated the feasibility of using this software for 2D and 3D simulations of HTS in different applications (transport current and applied external magnetic field). More detailed description of the numerical implementation in Flux 3D, including some other examples, can be found in [5] [6].

\section{A. FEM formulation}

We have employed the so called $\mathbf{T}-\mathbf{T}_{\mathbf{0}}-\boldsymbol{\Phi}$ formulation which uses the current vector potential $\mathbf{T}$ and the magnetic scalar potential $\mathbf{\Phi}$, defined as follows: $\nabla \times \mathbf{T}=\mathbf{J}$ and $\mathbf{H}=$ $\mathbf{T}-\nabla \boldsymbol{\Phi} . \mathbf{T}_{\mathbf{0}}$ is an auxiliary potential necessary for imposing a transport current to the superconductor [7].

Using the relation $\mathbf{B}=\mu_{0} \mathbf{H}$, Faraday's and Gauss's laws, which must be satisfied, are expressed as:

$$
\begin{aligned}
& \nabla \times \mathbf{E}+\frac{\partial}{\partial t}\left(\mu_{0}(\mathbf{T}-\nabla \boldsymbol{\Phi})\right)=0 \\
& \nabla \cdot\left(\mu_{0}(\mathbf{T}-\nabla \boldsymbol{\Phi})\right)=0
\end{aligned}
$$

The electrical behavior of the superconducting material is modeled by means of the following relations (see Fig. 3):

$$
\begin{cases}|\mathbf{E}|=\rho_{0}|\mathbf{J}| & \text { for }|\mathbf{J}| \leq J_{r} \\ |\mathbf{E}|=E_{0}\left(\frac{|\mathbf{J}|}{J_{c}}-1\right)^{n} & \text { for }|\mathbf{J}|>J_{r}\end{cases}
$$

where $E$ is the electric field, $J$ is the current density, $J_{c}$ is the critical current density (defined with the standard $1 \mu \mathrm{V} / \mathrm{cm}$ electric field criterion). $E_{0}=0.1 \mathrm{~V} / \mathrm{cm}$ and $n=3$ are the best fitting parameters of the $E-J$ law [1] [8].

Note that $E_{0}$ is not linked to the critical electric field $E_{c}$ and that $J_{c}$ has to be modified according to the scaling process (see section II-B below). The direction of $\mathbf{E}$ is given by the direction of $\mathbf{J}$.

Since $\left(|\mathbf{J}| / J_{c}-1\right)^{n}$ is not consistent for $|\mathbf{J}| \leq J_{c}$ the $E-J$ relation must be defined by pieces. The first part of equation (2) defines $E$ in sub-critical regime. The second part corresponds to over-critical regime, and is given by experimental $I-V$ data of YBCO thin films [8]. This equation fits better the experimental behavior of this material in overcritical regime than the traditionally used power-law written in the form $|\mathbf{E}|=E_{c}\left(|\mathbf{J}| / J_{c}\right)^{n}$.

Finally, for reasons of numerical stability of the solving algorithm and for avoiding division by zero, a residual resistivity $\rho_{0}=10^{-12} \Omega \cdot \mathrm{cm}$ is used for current density less or equal $J_{r}=1.0001 J_{c} . J_{r}$ is the value of $J$ where $\frac{\partial}{\partial J} E_{0}\left(\frac{J}{J_{c}}-1\right)^{n}=\rho_{0}$. The value of $\rho_{0}$ is chosen according to the criterion given in [5]. Its value has to be less than $10^{-2} E_{c} / J_{c}$. As the critical current density has been determined with the $1 \mu \mathrm{V} / \mathrm{cm}$ electric field, the value of $\rho_{0}$ has to be less than $3.3310^{-12} \Omega \cdot \mathrm{cm}$.

\section{B. Implemented FCL model}

A FEM simulation is considered to be optimal if the finite elements of the mesh are regular (all sides have roughly the same length). The geometric aspect ratio of this FCL is 11, 900 (5 mm length versus $420 \mathrm{~nm}$ total thickness) and requires a very fine mesh with a huge number of elements, which would result in an enormous consumption of CPU time and memory. Decreasing the finesse of the mesh, on the other hand, results in very extended elements along the width of the FCL, which leads to problems with the convergence of the algorithm, and consequently, to wrong results.

Therefore, to avoid this problem, we have multiplied the thickness of the layers by a factor ratio, which is equal to 1000 for the simulations presented in this paper. Using a lower factor (being closer to reality) requires considerably more computation resources.

Both the critical current $I_{c}$ of the YBCO layer and the resistance of the Au layer depend on the cross section, therefore on the thickness as well. That is why the following electric parameters have been modified as:

- $J_{c}=J_{c}^{*} /$ ratio

- $\rho_{\mathrm{Au}}=\rho_{\mathrm{Au}}^{*} \times$ ratio

$J_{c}$ is the value of the parameter "critical current density" and $\rho_{\mathrm{Au}}$ is the electrical resistivity used in the simulations whereas $J_{c}^{*}=3 \cdot 10^{6} \mathrm{~A} / \mathrm{cm}^{2}$ (defined with the standard $1 \mu \mathrm{V} / \mathrm{cm}$ electric field criterion) and $\rho_{\mathrm{Au}}^{*}=1 \cdot 10^{-6} \Omega \cdot \mathrm{cm}$ are the real critical current density of the YBCO material and the real resistivity of the Au layer. In this way, the YBCO critical current $I_{c}$ and the resistance of the Au layer do not change. Thus, the critical current $I_{c}$ of the simulated FCL is equal to 14.4 $\mathrm{A}$ as in the experimental device.

The FEM formulation (1) uses the magnetic scalar potential $\boldsymbol{\Phi}$. Changing the thicknesses of the layers leads to changing the inductive behavior of the simulated model. However the influence of the ratio value is limited by the fact that we are interested in over-critical regime (up to $3 I_{c}$ ). In this current range, the YBCO material has a resistive electrical behavior. Moreover, the resistivity of the gold layer has been adapted for having the same resistance than the real device, and we work at industrial frequencies (low frequency range) without external magnetic field applied. In this way, the local properties of the materials are essentially resistive, and the influence of the ratio value on the results can be considered as negligible. 


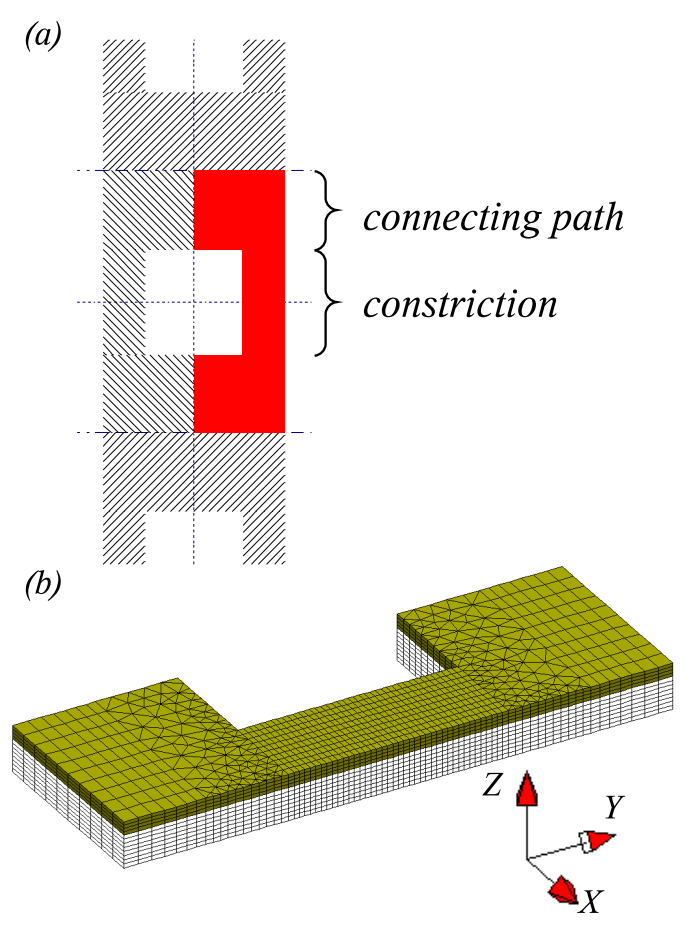

Fig. 4. Implemented geometry: (a) top view and (b) FEM mesh

Finally, the complexity of the problem is reduced by taking into account the periodicity along and the symmetry with respect to the $Y$ axis, see Fig. 4(a), which allows the simulation of only a part of the full geometry. Finally, Fig. 4(b) shows the implemented geometry with the FEM mesh.

There are in total around 35,000 nodes and 100,000 finite elements. For this geometry, a single simulation (2 cycles at $50 \mathrm{~Hz}$ ) with 300 time steps takes 3 days on a $3 \mathrm{GHz}$ Xeon PC for currents below $I_{c}$ and about 10 days for currents equal to $3 I_{c}$.

\section{RESULTS AND DISCUSSION}

\section{A. Results obtained with the original geometry}

For our simulations, we have used a driving sinusoidal current at $50 \mathrm{~Hz}$ with amplitude $3 I_{c}$ [1]. Figure 5 shows the current density and the electric field in the FCL with a current of $3 I_{c}$ at peak value (i.e. $\omega t=3 \pi / 2$ ). As the electrical terminals have been imposed on both the external YBCO and gold faces along the $Y$ axis, and with respect to the $X$ and $Y$ components, the $Z$ component of $\mathbf{J}$ and $\mathbf{E}$ is very low, and is not observable on these figures.

A high local electric field is observed in the region of the internal corner of the constriction, see Fig. 5(b). Because of the power law relation in (2), the instantaneous power losses (dot product between $E$ and $J$ ) are very high in this area and lead to significant local heating of the material.

In this way, the numerical simulations confirm the localization of the damage in the internal region of the constrictions because of the local heating. Therefore, we propose new geometries without right angles in the constrictions, which is expected to decrease the value of the instantaneous losses in these areas (more uniform repartition of the electric field) and

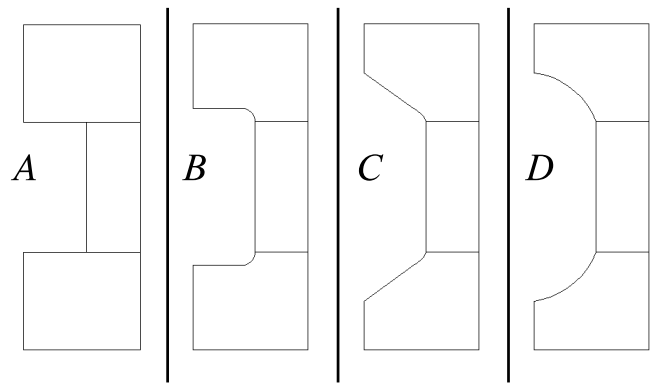

Fig. 6. Original (A) and proposed (B, C, D) geometries

to facilitate the cooling thanks to the larger surface (improved thermal exchange). These new geometries are represented in Fig. 6.

On Fig. 5(a), the current density along the external straight edge of the device is in the upper range of the scale. This is a consequence of a dimensional effect similar to the skin effect in massive conductors, which tends to force current to flow in the external part. The value of the skin depth for a linear conductor is equal to $\sqrt{2 \rho /\left(\omega \mu_{0} \mu_{r}\right)}$. At $4 J_{c}$, the resistivity $(E / J)$ of the YBCO material is equal to $2.2510^{-4} \Omega \cdot \mathrm{cm}$, which leads to a skin depth $e=120 \mu \mathrm{m}$ at $50 \mathrm{~Hz}$. This value is more than 6 times lower than the width of the constriction $(0.8 \mathrm{~mm})$. In the case of the real constriction, this effect is more pronounced $(e=4 \mu \mathrm{m})$ since the critical current density $J_{c}^{*}$ has been multiplied by 1000 according to section II-B.

\section{B. Results with the improved geometries}

Figure 7 shows the current density and the electric field in the FCL with a current of $3 I_{c}$ at peak value. Using geometries without right angles leads to significant decrease of the electric field and consequently of the local power losses in the internal corners of the constrictions. This can be seen by comparing Fig. 5(b) and 7(b). In this way, the risk of irreversible damage of the material due to overheating during fault event is going to be reduced.

There are different possible configurations, as illustrated in Fig. 6, therefore we have tried to select the optimal geometry, that is the one which leads to a decrease of the electric field in the corner of the constrictions and at the same time dissipates most in overcritical regime, without increasing the power losses in nominal regime (below $I_{c}$ ).

In order to find this geometry, we have compared the AC losses in sub-critical regime (at $0.8 I_{c}$ ), the power dissipation in over-critical regime (at $3 I_{c}$ ), shown in Fig. 8, and the maximum value of the electric field in the corner at peak current $(\omega t=3 \pi / 2)$, shown in Fig. 9.

The AC power losses (that is the integral of the dot product of $E$ and $J$ over a cycle) at $0.8 I_{c}$ are similar for the four geometries. There are in the order of $2 \cdot 10^{-4} \mathrm{~W} / \mathrm{cm}^{2}$. This value is in agreement with experimental data [1] and confirms the value chosen for $\rho_{0}$ (see section II-A).

On the contrary, the power dissipation in over-critical regime varies in the four geometries, see Fig. 8.

Geometries "C" and "D" turn out to be most advantageous because they allow a significant decrease (up to a factor 3 ) 


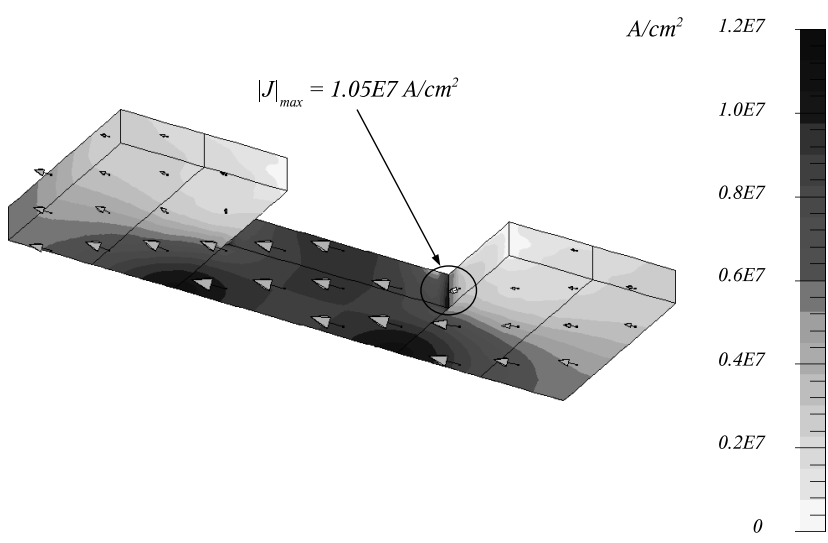

(a) Current density $|J|$

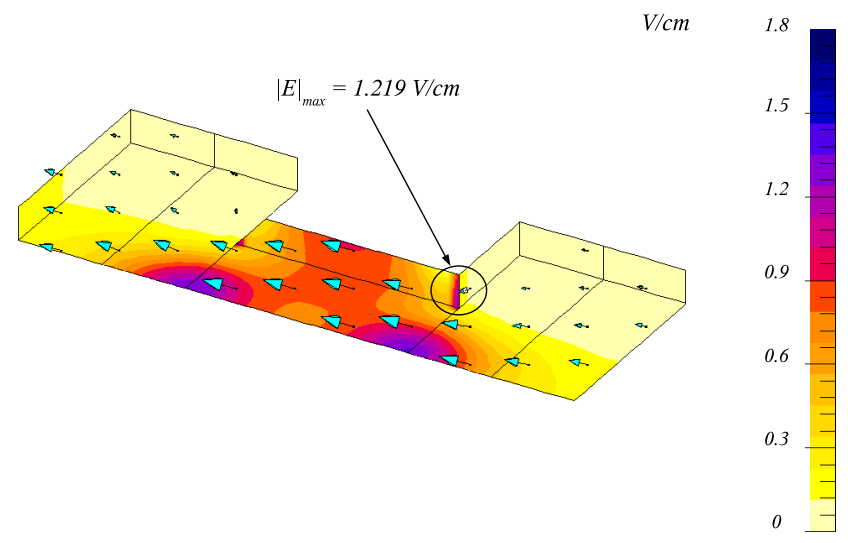

(b) Electrical field $|E|$

Fig. 5. Simulation results using a geometry with sharp corners (geometry "A" in Fig. 6 , YBCO only) with $I=3 I_{c}$ at $\omega t=3 \pi / 2\left(J_{c}^{*}=3 \cdot 10^{6} \mathrm{~A} / \mathrm{cm}^{2}\right)$

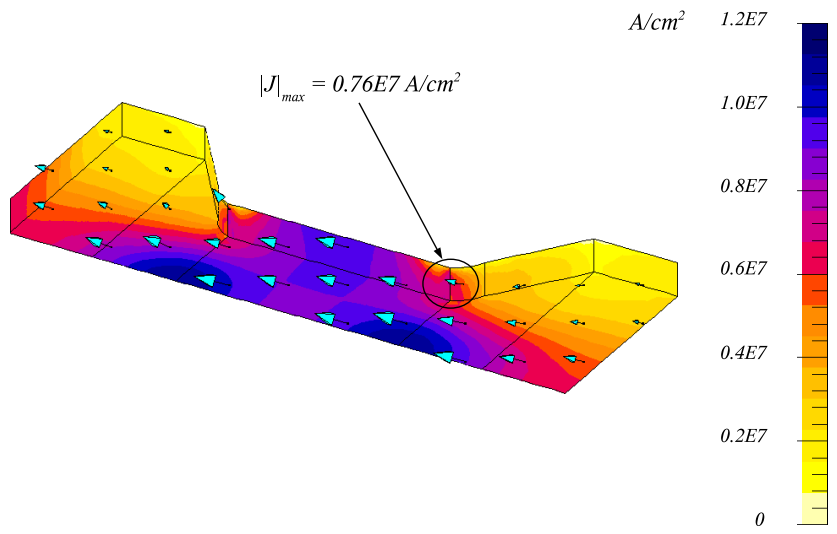

(a) Current density $|J|$

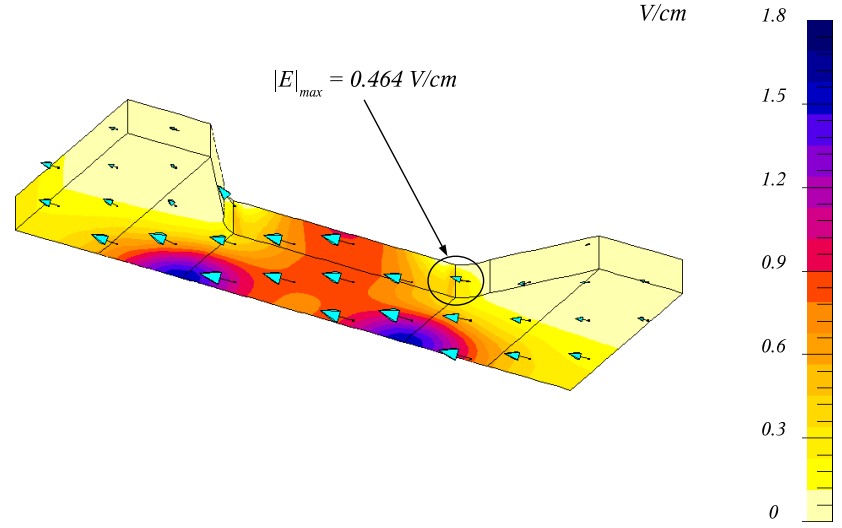

(b) Electrical field $|E|$

Fig. 7. Simulation results using a geometry without sharp corners (geometry " $\mathrm{C}^{\prime}$ in Fig. 6 , YBCO only) with $I=3 I_{c}$ at $\omega t=3 \pi / 2\left(J_{c}^{*}=3 \cdot 10^{6} \mathrm{~A} / \mathrm{cm}^{2}\right)$

of the electric field near the angle of the constrictions, see Fig. 9, and consequently, a strong reduction of the power dissipation in this area (less heating). In addition, compared to the geometry "A", the section of the connecting path is reduced, which forces the current to flow in already saturated zones, thus increasing the global power dissipation. This is an important advantage since the more power is absorbed in the FCL in over-critical regime, the more effective it is due to the higher impedance of the device during a fault event. Another important point is that a larger impedance implies a faster transition from nominal to limiting regime.

As far as the geometry "B" is concerned, slightly suppressing the right angles leads to a small decrease of the electric field, which results in a slight decrease of the total dissipated losses, see Fig. 8 and 9. In fact, rounding the right angles leads to a local reduction of the cross sections $S$ of the connecting path, which may be expected to increase the total power losses because of the increased resistance $(R=\rho l / S)$. In this case, however, the total dissipated power is reduced and not increased for $I>I_{c}$ since the local power losses in the angles are very important and they are significantly reduced because of the electric field reduction due to the rounding, so that the

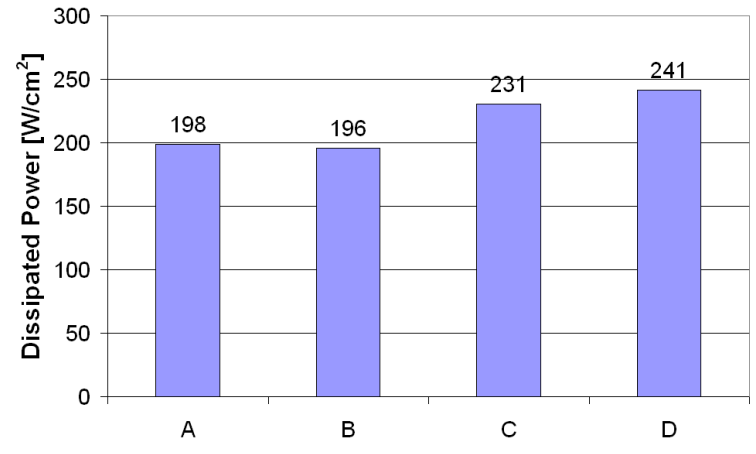

Fig. 8. Total power dissipation in the FCL for different geometries at $3 I_{C}$

total dissipation decreases, too.

\section{CONCLUSIONS AND FURTHER WORK}

We have used a 3D FEM modeling technique for novel simulations of $\mathrm{YBCO} / \mathrm{Au}$ thin film fault current limiters with particular attention to the mesh of the geometry because of the very large aspect ratio of the device. We have employed 


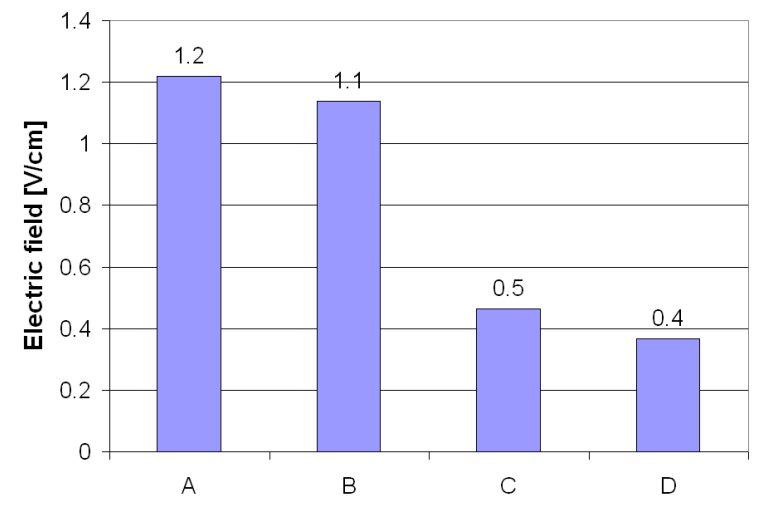

Fig. 9. Maximum electric field in the constriction's angle with $I=3 I_{c}$ at $\omega t=3 \pi / 2$

a specific $E-J$ power-law relation, more suitable for simulations of FCL because it fits well the experimental electrical behavior of YBCO materials.

We have demonstrated the importance of the FCL geometry for the optimization of the device, i.e. protection of the material against burning due to local heating, and also improved limitation because of higher power absorption during the limiting phase.

Two new geometries have been proposed in order to optimize the performance of the FCL. However, additional experimental testing is necessary in order to validate the suggested improvements. The implementation of a test wafer with the novel geometries is currently under way.

The next stage of this novel $3 \mathrm{D}$ modeling technique is to develop new FEM code for electromagnetic-thermal coupling in order to take into account the thermal behavior of the device, namely the $J_{c}(T)$ dependence and the propagation of quench.

\section{REFERENCES}

[1] M. Decroux, L. Antognazza, N. Musolino, E. de Chambrier, S. Reymond, J.-M. Triscone, Ø. Fischer, W. Paul, M. Chen, ’Properties of YBCO films at high current densities: Fault current limiter implications", IEEE Trans. Appl. Supercond., Vol. 11, pp 2046-2049, 2001.

[2] M. Lindmayer, H. Mosebach, "Current Limiting Properties of YBCOFilms on Sapphire Substrates", IEEE Trans. Appl. Supercond., Vol. 9, pp. 1369-1372, 1999.

[3] M. Decroux, L. Antognazza, S. Reymond, W. Paul, M. Chen, Ø. Fischer, "Studies of YBCO Strip Lines under Voltage Pulses: Optimisation of the Design of Fault Current Limiters", IEEE Trans. Appl. Supercond., Vol. 13, pp 1988-1991, 2003.

[4] CEDRAT S.A., Meylan, France, http://www.cedrat.com

[5] S. Stavrev, F. Grilli, B. Dutoit, N. Nibbio, E. Vinot, I. Klutsch, G. Meunier. P. Tixador, Y. Yang, E. Martinez, "Comparison of Numerical Methods for Modelling of Superconductors", IEEE Trans. Mag., Vol. 38, pp 849-852, 2002.

[6] F. Grilli, M. Costa Bouzo, Y. Yang, C. Beduz, B. Dutoit, "Finite element method analysis of the coupling effect between superconducting filaments of different aspect ratio", Supercond. Sci. Tech., Vol. 16, pp 1228-1234, 2003.

[7] G. Meunier, Y. Le Floch, C. Guérin, "A non linear circuit coupled T$T_{0}-\Phi$ formulation for solid conductors", IEEE Trans. Mag., Vol.39, pp 1729-1732, 2003.

[8] L. Antognazza, M. Decroux, S. Reymond, E. de Chambrier, J.-M. Triscone, W. Paul, M. Chen, Ø. Fischer, "Simulation of the behavior of superconducting YBCO lines at high current densities", Physica C372376, pp 1684-1687, 2002. 